Kompass

Dermatologie

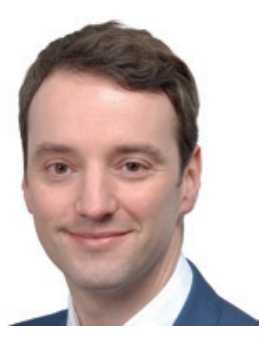

Max Schlaak

Klinik und Poliklinik für Dermatologie und Allergologie, München, Deutschland

Das letzte Jahrzehnt stand im Zeichen der praxisändernden Behandlungen beim fortgeschrittenen malignen Melanom. Durch die Entwicklung von zielgerichteten und Immuntherapien haben unsere metastasierten Patienten mittlerweile eine deutlich höhere Chance, länger mit dieser schweren Erkrankung zu leben.

Ich erinnere mich an die Anfänge vor ca. 10 Jahren mit dem Einsatz des Checkpoint-Inhibitors Ipilimumab. Die Therapie war neu und es fehlten größere Vorerfahrungen. Leider profitierte dann nur eine kleinere Gruppe von Patienten von der Therapie, diese dann aber häufig langfristig. Da wir Dermatologen mit die ersten waren, die diese Behandlungen einsetzten, profitieren wir nun von vielen Jahren an Erfahrung.

Mittlerweile werden anti-PD-1-Antikörper als Monotherapie und in Kombination mit Ipilimumab eingesetzt, mit deutlich höheren Ansprechraten. Diese Therapien stehen nicht nur im metastasierten Stadium, sondern auch adjuvant für Patienten mit Lymphknotenmetastasen, zur Verfügung. Auch der neoadjuvante Einsatz wird momentan evaluiert.

\title{
Aktuelle Errungenschaften der Immuntherapie beim fortgeschrittenen und metastasierten malignen Melanom
}

Die zurückliegenden Erfolge für unsere Patienten bedeuten aber nicht, dass wir nicht vor weiteren Herausforderungen stehen. Die Behandlung mit Checkpoint-Inhibitoren wirkt nicht bei jedem Patienten. In dieser Ausgabe des Kompass Dermatologie werden von Kollegen aus Mannheim und Heidelberg in einem Review die Resistenzmechanismen beschrieben und die Entwicklungen zur Verbesserung der Wirksamkeit zusammengefasst. Das Ziel wird sein, noch bessere, möglichst gut verträgliche Kombinationen zu finden. In Studien wurde zuletzt z.B. die Kombination von Immunund zielgerichteten Therapien untersucht. Ich kann mir vorstellen, dass wir in Zukunft auf mehr Patientencharakteristika und Tumoreigenschaften zurückgreifen können und so Therapien noch personalisierter geplant werden können.

Als kleinen Blick über den Tellerrand fassen Frau Wessely und Herr Heppt aus Erlangen die Wirksamkeit der Immuntherapien beim seltenen, aber häufig aggressiv verlaufenden, metastasierten Aderhautmelanom zusammen. Aufgrund der Biologie dieser Tumorerkrankung sind weitere Anstrengun- gen notwendig, um Patienten in Zukunft wirksamere Therapien anbieten zu können. Einen wichtigen Punkt in der Behandlung von Patienten mit Checkpoint-Inhibitoren stellt das Erkennen und Behandeln von $\mathrm{Ne}$ benwirkungen dar. Leider sind uns allen seltene und schwere Nebenwirkungen, die bei unseren Patienten auftraten, im Gedächtnis.

In der Arbeit von Herrn Eggermont wird nun beschrieben, dass das Auftreten von bestimmten Nebenwirkungen in der adjuvanten Behandlung des malignen Melanoms mit einem besseren Verlauf der Erkrankung einhergeht.

Ich hoffe, dass Ihnen das vorliegende Schwerpunktheft zum Thema DermatoOnkologie eine interessante Übersicht über die verschiedenen Facetten der Immuntherapie gibt und verbleibe mit kollegialen Grüßen

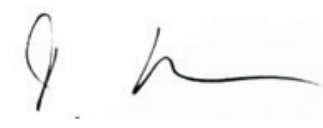

Ihr Max Schlaak 